\title{
Model structure on the category of small topological categories
}

\section{Amrani Ilias}

Received: 12 April 2012 / Accepted: 13 June 2013 / Published online: 27 June 2013

(C) Tbilisi Centre for Mathematical Sciences 2013

\begin{abstract}
We give a short proof of the existence of a cofibrantly generated Quillen model structure on the category of small topologically enriched categories, obtained by a transfer from Bergner's model structure on simplicially enriched categories.
\end{abstract}

Keywords Model categories $\cdot \infty$-Categories

Mathematics Subject Classification (2000) Primary $55 \cdot 18$

\section{Introduction}

We describe a Quillen model structure on the category of small topological categories. This model structure is obtained through a transfer from Bergner's model structure on the category of simplicial categories [4]. Bergner's original argument for the existence of a model structure on simplicial categories can be adapted to topological categories; this has been carried out in [1]. The proof to be presented here is much shorter, since it relies on an explicit construction of path-objects for topological categories. Recently, Berger-Moerdijk [3] gave a general existence result for a model structure on $\mathrm{V}$-enriched categories including the cases $\mathrm{V}=$ Top and $\mathrm{V}=\mathrm{sSet}$. We hope that our construction of path-objects for topological categories nevertheless presents some

Communicated by Jiri Rosicky.

Supported by the project CZ.1.07/2.3.00/20.0003 of the Operational Programme Education for Competitiveness of the Ministry of Education, Youth and Sports of the Czech Republic.

\footnotetext{
A. Ilias $(\varangle)$

Department of Mathematics, Masaryk University, Brno, Czech Republic

e-mail: ilias.amranifedotov@gmail.com; amrani@math.muni.cz
} 
interest. We implicitly use the fact that all topological categories are fibrant which is not the case in the simplicial setting.

\section{The model structure}

In this article, the category of compactly generated topological Hausdorff spaces will be denoted by Top, which is a simplicial symmetric monoidal closed model category. Before stating the main theorem we introduce some notations and definitions.

Remark 2.1 A topological category is a category enriched over Top. The category of all (small) topological categories is denoted by Cat Top. The morphisms in Cat Top $_{\text {are }}$ the enriched functors. It is a complete and cocomplete category. The category $\mathrm{V}$ will be either the category sSet or Top.

Definition 2.2 Let $\mathcal{U}: \mathrm{V} \rightarrow$ Catv be the functor defined as follows:

For each object $S \in \mathrm{V}, \mathcal{U}(S)$ is the enriched category with two objects $x$ and $y$ such that $\operatorname{Map}_{\mathcal{U}(S)}(x, y)=S$.

Definition 2.3 There is an obvious monoidal functor $\pi_{0}:$ Cat ${ }_{\text {Top }} \rightarrow$ Cat which sends a topological category to its homotopy category obtained by replacing the hom-spaces with their sets of path-components.

Theorem 2.4 The category Cat $_{\text {Top }}$ admits a cofibrantly generated model structure defined as follows.

Weak equivalences (Dwyer-Kan equivalences) F : C $\rightarrow$ D are those functors which satisfy the following conditions.

WT1: The morphism $\operatorname{Map}_{\mathrm{C}}(a, b) \rightarrow \operatorname{Map}_{\mathrm{D}}(\mathrm{F} a, \mathrm{~F} b)$ is a weak homotopy equivalence in the category Top.

WT2: The induced morphism $\pi_{0} \mathrm{~F}: \pi_{0} \mathrm{C} \rightarrow \pi_{0} \mathrm{D}$ is an equivalence in Cat, i.e. fully faithful and essentially surjective functor.

Fibrations are morphisms $\mathrm{F}: \mathrm{C} \rightarrow \mathrm{D}$ which satisfy:

FT1: The morphism $\operatorname{Map}_{\mathrm{C}}(a, b) \rightarrow \operatorname{Map}_{\mathrm{D}}(\mathrm{F} a, \mathrm{~F} b)$ is a fibration in Top.

FT2: For any objects $a \in \mathrm{C}$ and $b^{\prime} \in \mathrm{D}$, and any weak equivalence $e: \mathrm{F}(a) \rightarrow b^{\prime}$ in $\mathrm{D}$ (i.e., induces an isomorphism in $\left.\pi_{0} \mathrm{D}\right)$, there exists a weak equivalence $d: a \rightarrow b$ in $\mathrm{C}$ such that $\mathrm{F} d=e$.

The set I of generating cofibrations is given by:

CT1: $\left|\mathcal{U} \partial \Delta^{n}\right| \rightarrow\left|\mathcal{U} \Delta^{n}\right|$, for $n \geq 0$.

$C T 1: \emptyset \rightarrow\{x\}$, where $\emptyset$ is the empty topological category and $\{x\}$ is the category with one object and one morphism.

The set $J$ of generating acyclic cofibrations is given by:

ACT1: $\left|\mathcal{U} \Lambda_{i}^{n}\right| \rightarrow\left|\mathcal{U} \Delta^{n}\right|$, for $0 \leq n$ and $0 \leq i \leq n$.

ACT2: (Interval categories) $\{x\} \rightarrow|\mathcal{H}|$ where $\{\mathcal{H}\}$ runs through all countable interval categories in the sense of Bergner [4]. 
Moreover, the adjunction:

$$
\text { Cat }_{\text {sSet }} \stackrel{\mid-1}{\underset{\text { sing }}{\longleftrightarrow}} \text { Cat }_{\text {Top }}
$$

is a Quillen equivalence.

Proof The proof is given in Corollary 3.11.

Remark 2.5 The category Top with the standard model structure (i.e. equipped with weak homotopy equivalence and Serre fibrations) is Quillen equivalent to the standard model category of simplicial sets. The Quillen equivalence is given by the adjoint pair (geometric realization, singular functor). More precisely, a map of $f: X \rightarrow Y$ of topological spaces is a Serre fibration (resp. weak homotopy equivalence) if and only if $\operatorname{sing}(f)$ is a Kan fibration (resp. weak homotopy equivalence) [6, Proposition 4.5.26, Corollary 4.5.31]. Therefore, our main Theorem 2.4 can be understood as asserting the existence of a transfer of Bergner's model structure along the aforementioned adjunction, in the sense of $[2,2.6]$. The only point to check is that our condition FT2 corresponds to Bergner's condition FT2 under transfer, which is a straightforward verification.

Remark 2.6 Interval categories in the sense of Bergner [4] are $\mathrm{V}$-intervals in the sense of Berger-Moerdijk [3] for $V=$ sSet. Since all objects in Top are fibrant, a result of Berger-Moerdijk [3] implies that it is actually sufficient to choose a single interval category in ACT2.

\section{Proof of the main Theorem 2.4}

In this section, we give a proof of the existence of a cofibrantly model structure on

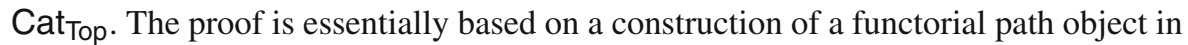
the category Cat Top. We take our inspiration from [5] and [7]. Let $I=[0,1]$, and $X$ a topological space. It is well known that the concatenation of paths (when it is possible) in the standard path object $X^{I}=\operatorname{map}(I, X)$ is not strictly associative. To solve this inconvenient problem we introduce the Moore path space.

Definition 3.1 Let $X$ be a topological space, and $\mathcal{M}(X)$ be the subspace of $X^{\mathbb{R}^{+}} \times \mathbb{R}^{+}$ with the following property:

$$
\mathcal{M}(X)=\left\{\left(\alpha: \mathbb{R}^{+} \rightarrow X, r\right) \in X^{\mathbb{R}^{+}} \times \mathbb{R}^{+} \mid \alpha(s)=\alpha(r), \forall s \geqslant r\right\} .
$$

The positive real number $r$ is called the length of the path. We denote by $\mathcal{M}^{r}(X)$ the space of paths of length $r$.

There is a natural inclusion of $X^{I}=\mathcal{M}^{1}(X) \rightarrow \mathcal{M}(X)$ wich is the inclusion of paths of length 1 . This inclusion is a weak equivalence. Moreover, we have two natural maps $\left(\delta_{-}, \delta_{+}\right): \mathcal{M}(X) \rightarrow X$ defined by $\delta_{-}(\alpha, r)=\alpha(0)$ and $\delta_{+}(\alpha, r)=\alpha(r)$. 
Lemma 3.2 The map $\left(\delta_{-}, \delta_{+}\right): \mathcal{M}(X) \rightarrow X \times X$ is a fibration in Top.

Proof Let $I^{n} \rightarrow I^{n+1}$ be a generating trivial cofibration in Top, and consider a commutative diagram:

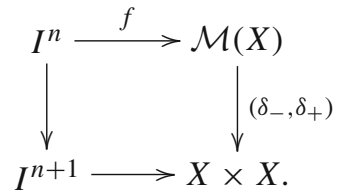

Since $I^{n}$ is compact, there exists $r \in \mathbb{R}^{+}$such that the image of $f$ lives in $\mathcal{M}^{r}(X) \subset$ $\mathcal{M}(X)$. Consequently, we have to construct a lifting for the following diagram:

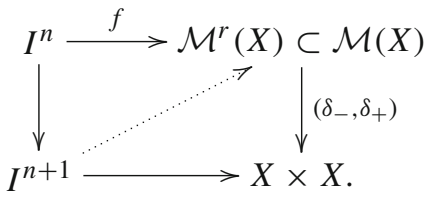

The existence of a lift is obvious, since $\mathcal{M}^{r}(X)$ is just a rescaling of $X^{I}$ by $r$.

Remark 3.3 The diagonal map $\Delta: X \rightarrow X \times X$ is factored by $X \rightarrow \mathcal{M}(X) \rightarrow$ $X \times X$, where the first map (weak equivalence) is the evident embedding of $X=$ $\mathcal{M}^{0}(X)$ (constant paths of length 0 ) in $\mathcal{M}(X)$. The second map is the fibration $\left(\delta_{-}, \delta_{+}\right)$ (Lemma 3.2).

Remark 3.4 Let $(\alpha, r)$ and $(\beta, s)$ two elements of $\mathcal{M}(X)$ such that $\alpha(r)=\beta(0)$, then we can define $(\beta * \alpha, r+s)$ by concatenation $*$. The composition law $*$ is strictly associative and unital when it is defined.

Definition 3.5 Let $\mathcal{P}:$ Cat $_{\text {Top }} \rightarrow$ Cat $_{\text {Top }}$ be a functor defined as follows. For any topological category $\mathrm{C}$, we declare:

(1) Objects of $\mathcal{P C}$ to be morphisms $a: x \stackrel{\sim}{\longrightarrow} y$ such that $a$ is a weak equivalence in $\mathrm{C}$, i.e., induces an isomorphism in $\pi_{0} \mathrm{C}$.

(2) The space of maps $\operatorname{Map}_{\mathcal{P C}}\left(x \rightarrow y, x^{\prime} \rightarrow y^{\prime}\right)$ between two objects $a: x \rightarrow y$ and $b: x^{\prime} \rightarrow y^{\prime}$ is given by the following pullback of the diagram in Top.

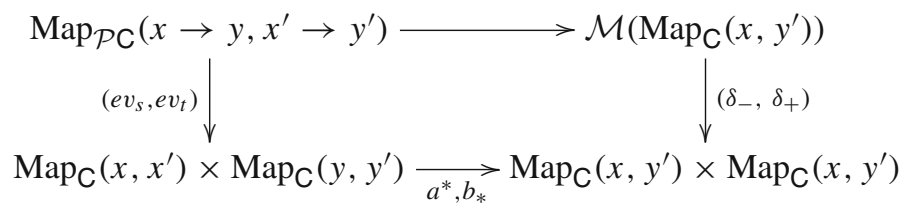

where $\mathcal{M}\left(\operatorname{Map}_{C}\left(x, y^{\prime}\right)\right)$ is the Moore path space associated to $\operatorname{Map}_{\mathrm{C}}\left(x, y^{\prime}\right)$ (Definition 3.1). 
Remark 3.6 The composition of morphisms in the category $\mathcal{P C}$ is explained in Sect. 3.1. In order to get an intuition about maps in the topological category $\mathcal{P C}$, we should remark that

$$
\operatorname{Map}_{\mathcal{P C}}\left(x \rightarrow y, x^{\prime} \rightarrow y^{\prime}\right)
$$

is a model for the homotopy pullback in Top of the diagram

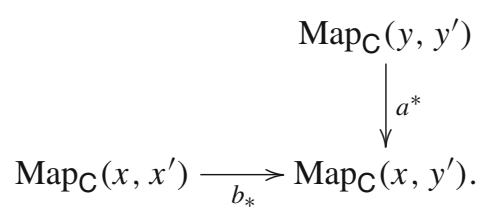

\subsection{Composition law in $\mathcal{P C}$}

Now, we define the composition law in the categorical path object introduced in Definition 3.5. Let $\mathrm{C}$ be a topological category. The categorical path object $\mathcal{P}(\mathrm{C})$ has as objects $a: x \rightarrow y$ where $a$ is a weak equivalence in $\mathrm{C}$. A morphism in $\mathcal{P}(\mathrm{C})$ between $a: x \rightarrow y$ and $b: x^{\prime} \rightarrow y^{\prime}$ is a diagram of the form

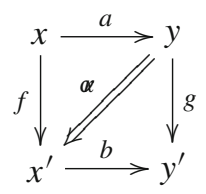

where $f, g$ are elements of $\operatorname{Map}_{\mathbf{C}}\left(x, x^{\prime}\right)$ and $\operatorname{Map}_{\mathrm{C}}\left(y, y^{\prime}\right)$, and $\alpha_{r}$ is an element of $\mathcal{M}\left(\operatorname{Map}_{\mathrm{C}}\left(x, y^{\prime}\right)\right)$ such that $\alpha_{r}$ is a homotopy (path) of length $r$ between $g . a$ and $b . f$. Let $a: x \rightarrow y, b: x^{\prime} \rightarrow y^{\prime}$ and $c: x^{\prime \prime} \rightarrow y^{\prime \prime}$ three objects of $\mathcal{P}(\mathrm{C})$. Consider the diagram

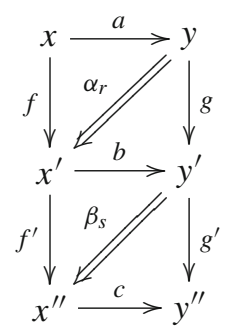

by composition we obtain 


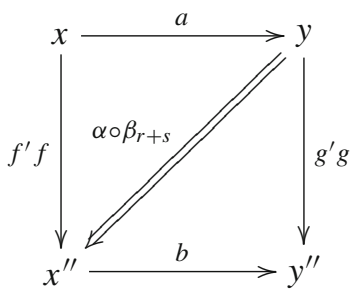

where $(\beta \circ \alpha)_{r+s} \in \mathcal{M}\left(\operatorname{Map}_{C}\left(x, y^{\prime \prime}\right)\right)$ is defined as the concatenation $\beta . f * g^{\prime} . \alpha$. The identity map $i d_{a}$ of $a: x \rightarrow y$ is given by $i d_{x}: x \rightarrow x, i d_{y}: y \rightarrow y$ and a constant Moore path of length 0 .

Lemma 3.7 The previous composition law is associative.

Proof Let take the following diagram:

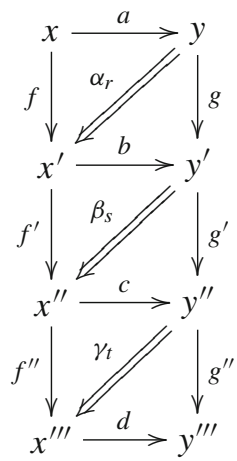

The composition of morphisms and Moore paths is strict and we get:

$$
\begin{aligned}
\left(\gamma_{t} \circ \beta_{s}\right) \circ \alpha_{r} & =\left(\gamma_{t} f^{\prime} f * g^{\prime \prime} \cdot \beta_{s}\right) \circ \alpha_{r} \\
& =\left(\gamma_{t} \cdot f^{\prime} * g^{\prime \prime} \cdot \beta_{s}\right) \cdot f * g^{\prime \prime} g^{\prime} \cdot \alpha_{r} \\
& =\gamma_{t} \cdot f^{\prime} f * g^{\prime \prime} \cdot \beta_{s} \cdot f * g^{\prime \prime} g^{\prime} \cdot \alpha_{r} \\
\gamma_{t} \circ\left(\beta_{s} \circ \alpha_{r}\right) & =\gamma_{t} \circ\left(\beta_{s} \cdot f * g^{\prime} \cdot \alpha_{r}\right) \\
& =\gamma_{t} \cdot f^{\prime} f * g^{\prime \prime} \cdot\left(\beta_{s} \cdot f * g^{\prime} \cdot \alpha_{r}\right) \\
& =\gamma_{t} \cdot f^{\prime} f * g^{\prime \prime} \cdot \beta_{s} \cdot f * g^{\prime \prime} g^{\prime} \cdot \alpha_{r}
\end{aligned}
$$

Remark 3.8 The diagonal map $\Delta: \mathrm{C} \rightarrow \mathrm{C} \times \mathrm{C}$ is factored as

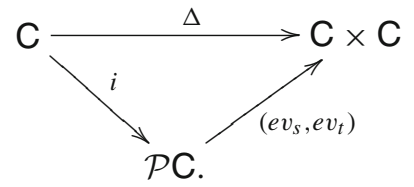


where

- $i: \mathrm{C} \rightarrow \mathcal{P C}$ is defined as $i(x)=i d_{x}: x \rightarrow x$.

- $e v_{s}(x \rightarrow y)=x$ and $e v_{t}(x \rightarrow y)=y$.

Lemma 3.9 The map $i: \mathrm{C} \rightarrow \mathcal{P C}$ is an equivalence of topological categories ( $c f$. Theorem 2.4).

Proof First, we check that $i$ is homotopically fully faithful i.e.,

$$
\operatorname{Map}_{\mathrm{C}}(x, y) \rightarrow \operatorname{Map}_{\mathcal{P C}}(i(x), i(y))
$$

is a weak equivalence of topological space, since by definition

$$
\operatorname{Map}_{\mathcal{P C}}(i(x), i(y))=\mathcal{M}\left(\operatorname{Map}_{\mathcal{C}}(x, y)\right) .
$$

For the homotopical essential surjectivity, lets take an object $a: x \rightarrow y$. By definition, there is a weak equivalence in $\mathcal{P C}$ between $i(a)$ and $a: x \rightarrow y$ given by

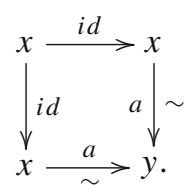

so $i$ is an equivalence of topological categories.

Lemma 3.10 Let $\mathrm{C}$ be a topological category, then the map $\left(e v_{s}, e v_{t}\right): \mathcal{P C} \rightarrow \mathrm{C}$ is a fibration in Cat $_{\text {Top }}(c f$. Theorem 2.4).

Proof First, we have to prove that

$$
\left(e v_{s}, e v_{t}\right): \operatorname{Map}_{\mathcal{P C}}\left(x \rightarrow y, x^{\prime} \rightarrow y^{\prime}\right) \rightarrow \operatorname{Map}_{\mathrm{C}}\left(x, x^{\prime}\right) \times \operatorname{Map}_{\mathrm{M}}\left(y, y^{\prime}\right)
$$

is a fibration of topological spaces. By definition $\left(e v_{s}, e v_{t}\right)$ is a pullback in Top of the fibration

$$
\left(\delta_{-}, \delta_{+}\right): \mathcal{M}\left(\operatorname{Map}_{\mathrm{C}}\left(x, y^{\prime}\right)\right) \rightarrow \operatorname{Map}_{\mathrm{C}}\left(x, y^{\prime}\right) \times \operatorname{Map}\left(x, y^{\prime}\right) .
$$

Second, let $w_{1}, w_{2}:(x, y) \rightarrow\left(x^{\prime}, y^{\prime}\right)$ be a weak equivalence in $\mathrm{C} \times \mathrm{C}$ such that there exists an object $a: x^{\prime} \rightarrow y^{\prime}$ in $\mathcal{P}$ C. Choose a homotopical inverse to $w_{2}$ denoted by $w_{2}^{-1}$ i.e., we have a path between $i d$ and $w_{2} w_{2}^{-1}$, and define the map $w_{2}^{-1} a w_{1}: x \rightarrow y$ which is also a weak homotopy equivalence. We remark, by definition, that we have a path

$$
\gamma:[0,1] \rightarrow \operatorname{Map}_{C}\left(x, y^{\prime}\right)
$$


between $w_{2} w_{2}^{-1} a w_{1}$ and $a w_{1}$. Therefore, we obtain a following morphism in $\mathcal{P C}$, namely:

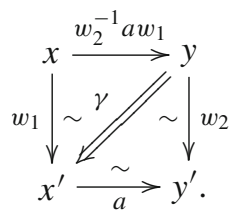

So we can lift the weak equivalence $\left(w_{1}, w_{2}\right) \in \mathrm{C} \times \mathrm{C}$ to a weak equivalence in $\mathcal{P C}$ given by $\left(w_{1}, w_{2}, \gamma\right)$.

Corollary 3.11 (Proof of Theorem 2.4) The category Cat Top $_{\text {is a cofibrantly generated }}$ model category and Quillen equivalent to the model category $\mathrm{Cat}_{\mathrm{sSet}}$.

Proof The standard Quillen adjunction (equivalence) between sSet and compactly generated topological spaces Top induces an adjunction between $\mathrm{Cat}_{\text {sSet }}$ and $\mathrm{Cat}_{\text {Top }}$. In order to apply the transfer principle [2, 2.6] we must first show that Quillen's small object argument is available. Since the domains of the generating (trivial) cofibrations in Top are compact, and hence $\otimes$-finite in the sense of [3, Lemma 2.15 in loc. cit], together with an adjunction argument establishes the required smallness of the generating (trivial) cofibrations of Cat Top. The existence of a transferred model structure on Cat $_{\text {Top }}$ is then a direct consequence of the Bergner's model structure on Cat ${ }_{\text {SSet }}$ [4] and the existence of path object (Lemmas 3.9,3.10). The Quillen equivalence between Cat $_{\text {Top }}$ and Cat $_{\text {sSet }}$ is obvious.

Acknowledgments The article is a part of my $\mathrm{PhD}$ thesis at Swiss Federal Institute of Technology, Lausanne (EPFL). I'm grateful to my advisor Kathryn Hess who made a big effort to read the manuscript in details. I'm also grateful to Clemens Berger for helpful comments on an earlier version of this note.

\section{References}

1. Amrani, I.: Categories simpliciales enrichies et K-Theorie de Waldhausen. PhD thesis (2010)

2. Berger, C., Moerdijk, I.: Axiomatic homotopy theory for operads. Comment. Math. Helvetici 78(4) (2003)

3. Berger, C., Moerdijk, I.: On the homotopy theory of enriched categories (preprint, arXiv:1201.2134, 2012)

4. Bergner, J.E.: A model category structure on the category of simplicial categories. Trans. Am. Math. Soc. 359(5), 2043 (2007)

5. Drinfeld, V.: Dg quotients of dg categories. J. Algebra 272(2), 643-691 (2004)

6. Fritsch, R., Piccinini, R.: Cellular Structures in Topology, vol. 19. Cambridge University Press, Cambridge (1990)

7. Tabuada, G.: A new quillen model for the Morita homotopy theory of dg categories. (arxiv, preprint math/0701205, 2007) 